\title{
Mesurer la communication? Ce que les outils de mesure font à la professionnalisation de la communication
}

Nicolas Peirot, ATER, Université de Bourgogne Franche-Comté, laboratoire CIMEOS, nicolas.peirot@univ-fcomte.fr

Sandrine Roginsky, Professeure, Université catholique de Louvain, laboratoire Lasco,

sandrine.roginsky@uclouvain.be 
Ce numéro thématique entend explorer les relations existantes entre mesures numériques et processus de professionnalisation des communicateurs.

Les métiers de la communication ne cessent de se diversifier et de se recomposer sous l'effet d'une technicisation croissante. Qu'il s'agisse de publicité, de relations presse ou publiques, de communication marketing ou managériale, ces activités apparaissent de plus en plus équipées numériquement. Les outils mobilisés sont le support d'un ensemble de pratiques de mesure de l'activité en ligne des internautes. Ils accompagnent l'injonction à rendre des comptes en termes de performance et de retour sur investissement imposée à la fonction communication (Lépine, 2013). Cette logique s'inscrit dans un processus de reconnaissance de la communication par la fonction managériale en termes d'efficacité et de rentabilité (Tixier, 1996). Cet idéal gestionnaire de mesure de la communication est d'ailleurs antérieur à la massification des technologies numériques. Différentes recherches témoignent ainsi des pratiques propres à l'étude des "publics » et à la mesure de « l'audience » des médias, qu'il s'agisse de presse écrite, de la radio ou de la télévision (Jouët, 2004 ; Wolton, 2003 ; Beaud, 1990). De même, le champ de la communication organisationnelle propose depuis plusieurs années un regard critique de la culture de la performance, des mesures et évaluations qui l'accompagnent (Lépine et Peyrelong, 2012; Gardère, 2012 ; Le Moënne et Parrini-Alemanno, 2010) ou encore des enjeux d'évaluation et de mesure de la réputation des entreprises (Boistel, 2014).

À travers ce numéro, il s'agit cependant d'acter une reconfiguration particulière $\mathrm{du}$ rapport entre mesure, organisation et professionnalisation dans un contexte de technicisation et de massification de la dimension numérique de la communication. En tant qu'innovation technologique, celle-ci se déploie à travers un imaginaire spécifique. Ce dernier apparaît marqué par différents discours d'accompagnement (Rebillard, 2011), inspirés de ceux du web « $2.0 »$ (O’Reilly, 2005), soulignant les vertus simplificatrices et transformatrices des dispositifs numériques. Selon les discours experts, nous assisterions à une extension du domaine du mesurable. Le calcul du « ROI digital » permettrait par exemple d'identifier les résultats directement imputables à une action de communication. Les dispositifs numériques de mesure diffusent ainsi la promesse d'une optimisation stratégique de la communication à travers l'analyse systématique des signaux (explicites) et traces (implicites) de navigation des internautes. La promesse d'efficacité des mesures numériques interroge ainsi directement les processus de construction de la reconnaissance chez les professionnel·les de la communication (Roginsky, 2018).

Les outils numériques de mesure que nous souhaitons mettre en discussion font entrer en jeu une réflexion plus générale autour du lien entre professionnalisation et numérique. Ce rapport est au cœur du débat public, drainant avec lui différentes problématiques héritées des premières révolutions industrielles. Bien que des travaux récents annoncent une fin possible du travail humain dans certaines sphères, notamment à travers l'automatisation des tâches (Frey et Osborne, 2013 ; OCDE, 2016), nous préférons nous détacher d'un questionnement trop large pour insister sur 
les réalités vécues par les acteurs professionnels de la communication. Loin de nier un phénomène d'automatisation numérique du travail, nous souhaitons comprendre plus précisément l'une de ses manifestations concrètes : les pratiques et idéaux de la mesure de la communication. Selon Albert Ogien $(2000,283)$, « mesurer est une activité qui consiste à produire une description numérique ». En cela, la mesure de la communication se trouve d'emblée au cœur d'une tension épistémologique qui interroge nécessairement les formes de légitimation professionnelle des communicateurs. En effet, parce qu'elle entend décrire numériquement, la mesure renvoie d'abord à une certaine conception "réaliste » de la communication. Celle-ci postule que " quelque chose de réel peut être "mesuré" » (Desrosières et Kott, 2005). En matière de communication, cette idée renvoie à différentes traditions théoriques (cybernétiques ou empirico-analytiques), largement mises à l'épreuve par les SIC, mais dans lesquelles les acteurs de la communication continuent possiblement à puiser, notamment pour répondre aux exigences gestionnaires. Cependant, en tant qu'activité de production de descriptions, la mesure de la communication ne peut évidemment pas être assimilée à une simple métrologie. Elle se présente aussi comme le résultat d'une série de conventions (Desrosières, 2001) liées à des dispositifs, des usages, des pratiques mais aussi des situations organisationnelles. Lorsqu'elle touche à la communication organisationnelle, la mesure interroge donc bien les modalités de légitimation professionnelle des communicateurs rices. D'un côté, une tentation bien connue, liée à la pensée gestionnaire, celle de chercher à asseoir la légitimité de la fonction communication sur une épistémologie positiviste. La mesure serait alors appréhendée comme une forme d'objectivation de la réalité de la communication (et souvent de ses " effets »). De l'autre côté, la mesure peut aussi renvoyer à une réalité construite. Cette idée n'est pas étrangère aux spécialistes des SIC et elle est aussi possiblement familière pour les communicateurs. La réalité complexe des dispositifs sociotechniques de mesure intègre donc aussi possiblement les pratiques et représentations des communicateurs. Le fait de mesurer la communication renvoie donc possiblement autant à son objectivation qu'à sa mise en discussion, ouvrant ainsi des voies différentes en matière de professionnalisation. Bien entendu, ces deux pôles sont heuristiques et n'entendent pas décrire des réalités figées. Il appartient ainsi aux articles de ce dossier de montrer la multitude des dépassements, cristallisations, ruptures et reconfigurations de cette tension dans un contexte de démocratisation et de massification des dispositifs de mesure numérique. Ainsi, c'est bien la diversité des appréhensions par les professionnel·les de la communication d'une mesure ambivalente que les articles de ce dossier interrogent. Quels sont les dispositifs de mesure qui accompagnent les communicateurs rices ? Quelles pratiques et à travers quelles injonctions se déploient-ils ? Comment s'articulent-ils aux formes d'évaluation bien connue des communicateurs rices ? Ces interrogations méritent également d'être posées à la lumière des «plateformes » (de réseaux socionumériques et/ou de notation) qui se multiplient et interrogent nécessairement la réalité professionnelle des communicateurs et communicatrices (Peirot et Galibert, 2019), que ce soit en 
tant que relais de leurs stratégies ou à l'inverse en tant que nouveaux intermédiaires s'imposant aux professionnel-les de la communication. Au-delà des usages et pratiques, c'est également la place de la mesure dans les savoirs professionnels des communicateurs ·rices qu'il s'agit d'interroger. Ainsi, peut-on trouver les traces d'une institutionnalisation des savoirs relatifs aux dispositifs numériques de mesure dans les corpus relatifs à la professionnalisation des communicateurs-rices (littérature spécialisée, descripteurs d'emploi, fiches métiers, fiches compétences, contenus pédagogiques de formations certifiantes et diplômantes) ? C'est à ces différentes questions que les articles du dossier tentent de répondre, que ce soit de façon croisée ou non, dans le cadre de monographies ou de propositions plus générales.

Ce numéro est ainsi structuré autour de deux axes. Le premier s'intéresse à la mesure comme forme de rationalité, en interrogeant ses conséquences sur la fonction communication. Il s'agit notamment d'aborder les enjeux de rationalisation gestionnaire et managériale qu'accompagnent les dispositifs et outils de mesure. Les articles de cet axe interrogent donc de façon systématique les liens qu'entretiennent mesure et évaluation de la communication, qu'il s'agisse des stratégies (dont on cherche à dire la valeur qu'elles auraient directement générée) ou de l'activité communicationnelle des salariés et internautes (dont on cherche à dire la valeur qu'elle génère). Les propositions, critiques, donnent ainsi à voir la permanence, les reconfigurations et les limites d'une mesure objectivante mise au service d'une norme « d'efficacité ».

L'article d'Aude Seurrat s'intéresse à la prescription de la mesure dans le cadre de formations professionnelles en communication et montre que l'évaluation par la mesure participe de l'institutionnalisation du modèle gestionnaire de la communication. Un tel mouvement introduit une réduction des phénomènes à du langage mathématique, à leur traduction en chiffres. En ce sens, comme le note Valérie Boussard (2009), l'évaluation peut être considérée comme un « fétichisme du chiffre (Bourdieu, 1964). Elle participe alors d'une neutralisation des enjeux qui ne se laissent pas appréhender par la mesure.

C'est aussi la prescription de la mesure, ici dans le domaine plus spécifique de la communication interne, que l'article de Thomas Heller explore. Là encore, la mesure est au service de l'impératif d'évaluation ; ce qui n'est d'ailleurs pas sans rappeler les travaux d'Albert Ogien qui montrent que la technique d'évaluation a également gagné, « à grands renforts de communication interne », l'univers des administrations publiques. Selon le sociologue, « il ne semble plus exister aujourd'hui de secteur de la vie sociale qui échappe encore à l'emprise de l'évaluation » $(2013,9)$. Or, Thomas Heller met en lumière deux limites de l'évaluation de la communication interne : une limite de type gestionnaire centrée sur les rapports de causalité entre communication et efficacité et une limite de type idéologico-politique centrée sur le rôle affiché de la communication interne dans la littérature professionnelle.

Mesurer pour évaluer, c'est aussi un leitmotiv dans le travail des gestionnaires de réseaux sociaux d'entreprises que Camille Imhoff étudie. L'évaluation permet ici 
de contrôler et de valoriser la participation, et ce faisant d'évaluer, de manière certes indirecte, les collaborateurs - ce qui peut d'ailleurs expliquer la réticence des salariés devant le déploiement de ce type de dispositifs. La mesure constitue alors bien un enjeu de professionnalisation de la communication et participe à l'affirmation de l'animateur de communautés au sein des organisations. L'article de Camille Imhoff met néanmoins en relief un paradoxe central : «l'ambition de la mesure » ne semble pas s'accompagner du développement de véritables outils de mesure.

C'est justement le développement d'un outil de mesure de la communication interne, dépassant certaines des limites mises en évidence dans les articles précédents, que l'article de Déborah Horlait et François Lambotte tente de proposer. Dans le cadre d'une recherche-action, il s'agit ainsi de problématiser la «mesurabilité de la communication interne » et plus particulièrement d'interroger les critères de la mesure. L'approche proposée montre que cette question pose directement celle de la définition de la communication interne. La mesure agit alors moins comme le relais d'une objectivation de la fonction communication que comme un révélateur des multiples dimensions de la communication interne. À ce titre, l'article souligne bien la grande diversité des approches existantes et finalement l'intérêt toujours renouvelé des professionnels pour la mesure de la communication interne.

Les deux articles qui suivent portent davantage sur les effets performatifs des mesures. Il s'agit donc de comprendre ce que celles-ci font exister et contribuent à former (et donc ce qu'elles donnent à voir sur ce qui compte en matière de communication). Il s'agit aussi et surtout de saisir en quoi les mesures répondent à des enjeux de légitimation professionnelle. En cela, ils s'inscrivent dans le deuxième axe de notre appel à articles, qui vise à questionner la reconnaissance professionnelle à l'aune du développement des dispositifs de mesure. Comment la culture numérique qui les accompagnent soutient-elle la reconnaissance de professions traditionnelles ou émergentes dans le secteur de la communication ? Comment ces dispositifs numériques de mesure participent-ils possiblement à libérer les communicateurs rices de certaines tâches, à reconnaître le travail effectué, à le valoriser, et peut-être à transformer effectivement le rapport des communicateurs à leur organisation voire au travail plus généralement? Comment les dispositifs de mesure interrogent-ils d'une manière générale les contours de «l'expertise communicationnelle » (Grignon, 2015)? Finalement, les outils de mesure participent-ils à requalifier la performance dans le domaine de la communication et, par ricochet, les activités des communicateurs ? Loin de s'opposer aux questions posées dans le premier axe, les articles présentés ici appellent au contraire à mettre en discussion la place qu'occupent les outils et dispositifs de mesure dans la professionnalisation des communicateurs rices ; notamment vis-à-vis d'une possible institutionnalisation des formes de légitimations présentées dans ces articles.

L'article de Camille Alloing et Julien Pierre analyse ainsi les « métriques numériques » par la manière dont elles sont manipulées, discutées ou critiquées par les praticien·nes. Les auteurs montrent que les métriques numériques n'ont de valeur 
que par la manière dont elles circulent dans l'organisation et dont elles s'agencent avec d'autres instruments de gestion ou dispositifs d'évaluation. Pour les community managers, il semblerait qu'elles aient avant tout pour fonction d'affirmer leur rôle d'interface entre leurs organisations et les publics plus que de diriger leurs actions.

Un élément que l'on retrouve dans l'article de Daniel Pélissier qui, à partir de l'analyse d'un exemple de mesure dans le contexte de la communication de recrutement, montre en quoi celle-ci participe de la stratégie des professionnels du secteur bancaire d'avoir un rôle d'intermédiation. L'article met également en avant les représentations associées aux mesures affichées dans les dispositifs et montre que la fiabilité de ces mesures est discutée par les jeunes diplômés. Autrement dit, il existe une variété d'interprétations des mesures.

Sans entrer dans l'exploration des conventions qui déterminent et régulent l'activité de mesure, dans leurs articles respectifs, Camille Alloing et Julien Pierre comme Daniel Pélissier renseignent par ailleurs sur le rôle joué par les dispositifs web dans la construction de mesures relatives à des activités de communication. Enfin, le dernier article du dossier nous amène au Québec pour comprendre la place accordée à la compréhension des mesures associées aux actions de communication dans les formations québécoises en communication numérique. Cette démarche fait écho à celle d'Aude Seurrat dans son travail sur la prescription de la mesure de la communication dans la formation professionnelle en France mais cette fois en s'intéressant aux programmes et contenus de cours que l'on trouve dans les institutions québécoises à destination d'un public étudiant. Alexandre Coutant et Marie-Danielle Tremblay montrent que la facilité d'accès aux mesures de la communication ne s'accompagne pas d'une formation à leur usage réfléchi. Ce qui les amène à conclure à la nécessité d'une formation plus solide sur cette question.

Les articles qui composent ce dossier offrent autant d'angles d'approche à travers lesquels nous souhaitons pouvoir mettre en lumière les différences et ressemblances relatives à la diffusion de ces outils, dispositifs et technologies de mesure de la communication mais aussi les conceptions, voire les croyances, qui les accompagnent dans un contexte de montée en puissance des plateformes numériques.

\section{Bibliographie}

Beaud, P. (1990). Éditorial. Réseaux, 39(1), 5.

Boistel, P. (2014). Réputation : un concept à définir, Communication et Organisation, 46, 211-224. doi : https://doi.org/10.4000/communicationorganisation.4796

Bourdieu, P. (1964). Travail et travailleurs en Algérie, Paris, France : Mouton.

Boussard, V. (2009). L'incontournable évaluation des performances individuelles : entre l'invention d'un modèle idéologique et la diffusion de dispositifs pratiques. Nouvelle Revue de psychosociologie, 2(8), 37-52. 
doi : https://doi.org/10.3917/nrp.008.0037

Desrosières, A. (2001). Entre réalisme métrologique et conventions d'équivalence : les ambiguïtés de la sociologie quantitative. Genèses, A(43), 112-127. doi : https://doi.org/10.3917/gen.043.0112

Desrosières, A. (2008). Gouverner par les nombres. L'argument statistique II. Paris, France : Presses de l'École des Mines de Paris.

Desrosières, A. et Kott, S. (2005). Quantifier. Genèses, 1(58), 2-3. doi : https://doi.org/10.3917/gen.058.0002

Frey, C. B. et Osborne M. A. (2013, septembre). The Future of Employment: How Susceptible are Jobs to Computerisation? PDF repéré à https://frama.link/xR8Ut3W1

Gardère, É. (2012). Institution communicante et nouveau management public. Communication et Organisation, 41, 27-39. doi : https://doi.org/10.4000/communicationorganisation.3706

Grignon (2015). L'expertise communicationnelle au prisme de ses instruments. L'exemple de Google Analytics. Communication \& Professionnalisation, 3, $23-$ 47. doi : https://doi.org/10.14428/rcompro.vi3.443

Jouët, J. (2004). Les dispositifs de construction de l'internaute par les mesures d'audience. Le Temps des médias, 3(2), 160-174. doi : https://doi.org/10.3917/tdm.003.0160

Le Moënne, C. et Parrini-Alemanno, S. (2010). Management de l'évaluation et communication. Communication et Organisation, 38, 7-14. URL : http://journals.openedition.org/communicationorganisation/1365

Lépine, V. (2013). L'évaluation de la communication : représentations et enjeux pour les professionnels. Cahiers du Resiproc, 1, 70-89. doi : https://doi.org/10.14428/rcompro.vi1.253

Lépine, V. et Peyrelong, M.-F. (2012). Aux frontières de deux métiers : les compétences communicationnelles en jeu dans les bibliothèques. Communication et Organisation, 41, 63-73. doi : https://doi.org/10.4000/communicationorganisation.3730

OCDE (2016, mai). Automatisation et travail indépendant dans une économie numérique. http://www.oecd.org/employment/future-of-work/

Ogien, A. (2000). La volonté de quantifier. Conceptions de la mesure de l'activité médicale. Annales. Histoires, Sciences Sociales, 55(2), 283-312. doi : https://doi.org/10.3406/ahess.2000.279847 
Ogien, A. (2013). Désacraliser le chiffre dans l'évaluation du secteur public. Versailles : Éditions Quae.

O'Reilly, T. (2005, 30 septembre). What Is Web 2.0. Design Patterns and Business Models for the Next Generation of Software.

http://www.oreilly.com/pub/a/web2/archive/what-is-web-20.html

Peirot, N. et Galibert, O. (2019). Entre communauté et multitude : une approche communicationnelle de l'instrumentalisation de la reconnaissance au sein de l'économie collaborative. Communication et Organisation, 55, 24-40. doi : https://doi.org/10.4000/communicationorganisation.7668

Rebillard, F. (2011). Du Web 2.0 au Web2 : fortunes et infortunes des discours d'accompagnement des réseaux socionumériques. Hermès, La Revue, 59, 25-30. doi : https://doi.org/10.4267/2042/45331

Roginsky, S. (2018). Les communicateurs de l'Europe : des acteurs frontières confrontés à l'hybridité professionnelle et organisationnelle. Communication \& Professionalisation, 7, 49-75. doi : https://doi.org/10.14428/rcompro.v7i1.18193

Tixier, M. (1996). Les outils de mesure de la communication : Regard critique sur l'état de l'art. Communication et Organisation, 10.

doi : https://doi.org/10.4000/communicationorganisation.1887

Wolton, D. (2003). Avant-propos : Audience et publics : économie, culture, politique. Hermès, La Revue, 37(3), 27-34. doi : https://doi.org/10.4267/2042/9382 